\title{
Building Companionship through Human-Robot Collaboration
}

\author{
Yanan $\mathrm{Li}^{1}$, Keng Peng Tee ${ }^{2}$, Shuzhi Sam $\mathrm{Ge}^{1}$, and Haizhou $\mathrm{Li}^{2}$ \\ 1 Social Robotics Laboratory (SRL), Interactive Digital Media Institute, and \\ Department of Electrical and Computer Engineering, National University of \\ Singapore, Singapore, 117576 \\ 2 Institute for Infocomm Research $\left(\mathrm{I}^{2} \mathrm{R}\right)$, Agency for Science, Technology and \\ Research, Singapore, 138632
}

\begin{abstract}
While human-robot collaboration has been studied intensively in the literature, little attention has been given to understanding the role of collaborative endeavours on enhancing the companionship between humans and robots. In this position paper, we explore the possibilities of building the human-robot companionship through collaborative activities. The design guideline of a companion robot Nancy developed at SRL is introduced, and preliminary studies on human-robot collaboration conducted at SRL and $\mathrm{I}^{2} \mathrm{R}$ are elaborated. Critical issues and technical challenges in human-robot collaboration systems are discussed. Through these discussions, we aim to draw the attention of the social robotics community to the importance of human-robot collaboration in companionship building, and stimulate more research effort in this emerging area.
\end{abstract}

\section{Introduction}

Population ageing has become an extremely serious issue affecting almost every country across the globe. The increasing shortfall of caregivers, together with the increasing occurrences of elderly disability, loneliness and depression, can put a strain on a country's healthcare and economic system, as well as create deep social problems. It is thus envisaged to be highly beneficial to create intelligent robots that provide companionship to ease loneliness and prevent depression.

Most works on robot companionship take an approach focusing on communications and interactions, such as providing cognitive support, personalized chats, or pet-like affective therapy. To expand the application scope and fulfil more general needs, research in robot companions should also look into goal-oriented collaborative activities between human and robot. This is motivated by the observation that people who work well together enjoy good bonding experience. Likewise, participating in a joint activity with a robot to achieve a practical goal (e.g. house cleaning) can increase feelings of companionship in the human towards the robot. At the same time, the robot fulfils its role to assist with activities of daily living, and due to its collaborative nature, imparts a sense of empowerment and personal autonomy to the human since he/she still needs to 
be actively involved in the joint task instead of just being a passive beneficiary of robot assistance.

In fact, robots still need human help to operate in unstructured environments like homes, since human beings and robots have complementary advantages. On the one hand, robots equipped with the state-of-art sensors, computing power, and powerful actuators are capable of carrying out repetitive, high-load tasks tirelessly and with high precision. On the other hand, human beings possess excellent cognitive skills and are generally superior in situational awareness and decision making. An effective human-robot collaboration system will free human beings from heavy loads and achieve more robust operability in unstructured environments by maximizing complementary capabilities of human beings and robots. In the context of elderly companionship, an added benefit for the elderly person comes from the cognitive stimulation associated with learning how to interact and work with a robot with an intelligence of its own.

While human-robot collaboration has been studied intensively in applications such as search and rescue, healthcare, and construction (see the surveys [1, $2]$ ), little attention has been given to understanding the role of collaborative endeavours on enhancing feelings of acceptance and companionship towards robots. Our position is that there is a strong fundamental link between collaboration and companionship, which is worth investigating to unlock new but pragmatic ways of alleviating loneliness and accomplishing tasks at hand. But first, technical challenges must be solved to enable human and robots to work together organically. Designing such a system requires interdisciplinary studies of robot design, sensor fusion, control design, and system integration, among others. Much effort has been made in the literature. However, in many situations robots are still not "intelligent" enough to collaborate with human beings in a natural and effective way. What are the reasons that have caused such a gap, and what can we do to fill it? In this position paper, we will attempt to shed light on key aspects of this question by discussing critical technical challenges in human-robot collaboration and results of preliminary studies conducted at SRL and $\mathrm{I}^{2} \mathrm{R}$. We aim to draw the attention of the social robotics community to the importance and yet-tobe-realized value of human-robot collaboration in companionship building, and stimulate more research effort from the community in this emerging area.

\section{Robot Companion Nancy for Human-Robot Collaboration}

A social robot named Nancy has been developed (Fig. 1) as a platform for investigations and experiments in human-robot collaboration and its role in building companionship. Details of the robot design can be found in [3]. Here, we outline how Nancy was designed to be suitable for human-robot collaboration.

\subsection{Design}

The overarching design guideline for Nancy has been to make it able to move in a human-like manner with high performance, flexibility, and safety. As shown 


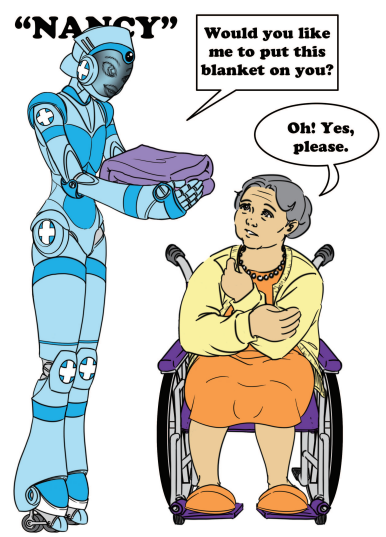

(a)

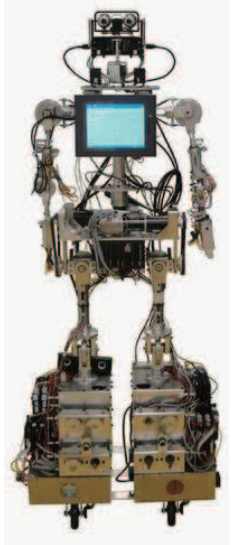

(b)

Fig. 1. (a) An envisaged scenario for Nancy the companion robot. (b) An actual first prototype of Nancy.

in Fig. 1, Nancy is a humanoid $160.7 \mathrm{~cm}$ in height and has a total of 32 degreesof-freedom (DOFs) throughout the whole body. Similar to a human, the upper body of Nancy includes the modules of hand, wrist, forearm, elbow, upper arm, shoulder, scapula, neck, head and torso. In particular, each arm has 8 DOFs to achieve human-like reaching and manipulating motions, and it has a 2-DOF neck and 3-DOF eyes with built-in cameras. A cable-pulley driving mechanism has been developed to ensure a light-weight upper body and to provide more natural physical human-robot interaction. Nancy's lower body has been designed to facilitate smooth and multi-direction movements with two legs and a wheel base.

\subsection{Sensing and Perception: Visual, Vocal and Haptic}

Nancy has been equipped with an intelligent scene-understanding engine to sense and perceive the environments through computer vision [4]. It is able to track and recognize objects, perceive the emotion, feeling and attitude of the human beings, and determine its attention autonomously in a scene. Another essential modality for human-robot collaboration is speech. Perception modules for recognition and understanding of spontaneous speech have been embedded in Nancy [3]. Besides vision and speech, haptic sensing and perception have also been built into the robot to enable studies in physical human-robot collaboration. In particular, force feedback is measured by a 6 -axis force/torque sensor mounted on Nancy's wrist, enabling human motion intention to be estimated for the purpose of human-robot co-manipulation [5]. Artificial skin has also been integrated to provide distributed tactile sensing to the robot as well as to enhance tactile sensation for the user [6]. 


\section{Technical Challenges in Human-Robot Collaboration}

Building a suitable robotic platform for human-robot collaboration is only half the story. More critical is the development of robotic intelligence that controls the robot's perception, behavior and joint action in the presence of the user. This section analyzes three key issues that are critical to improve the performance of a human-robot collaboration. One can see that there are a number of open problems that need to be solved by the social robotics community in order to achieve more natural and effective human-robot collaboration.

\subsection{Task Objective}

When designing a human-robot collaboration system, the first thing to come up with is the task objective, or, what is the intended outcome of the collaboration? Since a human-robot collaboration system includes two agents: a human being and a robot, this objective should well describe the coherence and conflict of individual objectives of two agents. This can be a specific application of multiagent systems [7], where the weight of the individual objective of the human being is larger than that of the robot in most cases. Our previous work [5] considered human-robot co-manipulation where the objective is to physically free the human from the heavy load. To prescribe an objective for a human-robot collaboration system is vital, but it can be challenging since the collaboration is dynamic. The human may change his/her strategy for the task, so the robot needs to be able to detect the excursion from the original plan and infer the new objective, or there should be an easy and intuitive way for the human to specify the task objective on-the-fly. It may also be essential to have prior knowledge of the physical limits of both human and robot, as well as models of the operation process and environment.

\subsection{Task Allocation and Adaptation}

After the objective is determined, the task needs to be allocated to the human and robot. Task allocation is based on the task objective and determined by analyzing the complementary advantages of human and robot. As discussed in Introduction, humans are generally better at understanding the circumstances and making decisions, and robots at carrying out regular tasks with the guaranteed performance. Therefore, it is natural to develop a leader-follower model, where the human being plays the role of a leader and executes a higher-level task. The leader-follower model has been employed in a vast number of research studies. In our previous work [5], we have shown that the robot becomes a load to the human being when the motion intention of the human being changes, and it can be estimated to allocate a larger share of the task to the robot. However, even if the motion intention of the human being can be predicted by the robot, the ideal case is that the robot follows the human "perfectly". We assume implicitly that the motion intention of the human being is stationary with respect 
to the actual robot trajectory. In other words, the adaptation of the robot trajectory is assumed to have no effect on the motion intention of the human being. However, human motion is also an output of the neuromuscular control system, so the dynamic interaction with the robot could well result in concurrent adaptation in the motion intention of the human being. This concurrent adaptation should be carefully investigated to ensure that the human-robot collaboration is stable.

Through learning and communication, the robot is able to increase its knowledge about the environment and task. Based on the acquired knowledge, it is possible for the robot to be allocated a higher level task. In robot programming by demonstration (see e.g. [8]), the human programs a robot to perform a certain complex task by physically holding a robot arm. After several iterations of operations, the robot is able to complete the task with less interference from the human being. In this simple example, the task allocation to the human being becomes less when the robot acquires more knowledge. It can be expected to develop a mechanism of adaptive task allocation, such that the proportion of the task allocation of the human being is increased only when necessary.

\subsection{Communication and Interface}

The communication between the human being and robot is important during the task execution. It can be used to resolve the conflict, disambiguate uncertainties, refine the task allocation and even the task objective. It relies on the sensing and perception capabilities of both human and robot. In most cases, communication is limited on the robot side due to limited sensing and perception capabilities, and this is the original motivation of human-robot collaboration. Technologies such as intention estimation [5], speech recognition [9], human detection and tracking $[10,11]$, and gesture recognition $[12,13]$ have been developed to enhance the perception capability of the robot. Augmented reality has been studied to make the human being and robot share the same reference frame [1]. Besides these, many sensors have been designed and developed for human-robot collaboration systems. Human beings understand the circumstances by listening, looking, smelling, and touching. It is natural to expect that robots have equivalent capabilities so a natural and efficient collaboration can be achieved. Nevertheless, most of state-of-art human-robot collaboration systems are embedded with only one or two of the above modules/technologies. A natural and efficient collaboration can be anticipated only when these modules/technologies are integrated to form a multi-modality interface. Such an interface goes beyond straightforward system integration, as sensor fusion needs to be carefully investigated and hierarchical architecture designed to handle differing levels of complexity in perception.

Many state-of-art human-robot collaboration systems are designed to make the robot understand the human being, while very few works in the literature address the issue of mutual understanding. On the one hand, there is still a long way to go for the robot to "understand" the human being, due to the limitation of sensing and perception capabilities, and learning skills. On the 
other hand, it is important to consider the acceptance and understanding of the robot by the human, especially when the human is a non-expert in robotics. For example, transparency and intuitive interface are essential in tele-operation. We have developed human-aided grasp assistance for easy and fast communication of grasp intent to robot [14] as well as robot-assisted grasping based on local proximity sensors to simplify the tele-operation task for the user [15]. A robot will be better understood and accepted if its behavior emulates that of human beings [1], and this can be taken into account when developing an interface for a human-robot collaboration system.

\section{Conclusion}

In this position paper, we have postulated and explored the intriguing possibility of building human-robot companionship through collaborative activities. Preliminary studies on human-robot interaction and collaboration conducted at SRL and $I^{2} R$ show promising results in terms of individual components such as motion intention estimation, human detection and tracking, speech and gesture recognition. However, much more work needs to be done, especially in integrating the various components into a stable, robust human-robot collaboration system, as well as designing and carrying out experimental trials to investigate the effect on human-robot companionship. Key issues in human-robot collaboration, including i) task objective, ii) task allocation and adaptation, and iii) communication and interface, have been discussed, and technical challenging problems proposed: adaptive task allocation, concurrent adaptation, mutual understanding, and multi-modality interface. More effective and natural human-robot collaboration leading to stronger companionship can be expected by resolving these problems. We see this topic as providing abundant scope for multi-disciplinary, collaborative research in social robotics.

\section{References}

1. S. A. Green, M. Billinghurst, X. Chen, and J. G. Chase, "Human-robot collaboration: A literature review and augmented reality approach in design," International Journal of Advanced Robotic Systems, vol. 5, no. 1, pp. 1-18, 2008.

2. A. Bauer, D. Wollherr, and M. Buss, "Human-robot collaboration: A survey," International Journal of Humanoid Robotics, vol. 5, no. 4, pp. 47-66, 2008.

3. S. S. Ge, J. J. Cabibihan, Z. Zhang, Y. Li, C. Meng, H. He, M. R. Safizadeh, Y. B. $\mathrm{Li}$, and J. Yang, "Design and development of nancy, a social robot," in Proceedings of the 8th International Conference on Ubiquitous Robots and Ambient Intelligence, (Incheon, Korea), pp. 568-573, 23-26, November 2011.

4. H. He, S. S. Ge, and Z. Zhang, "Visual attention prediction using saliency determination of scene understanding for social robots," International Journal of Social Robotics, vol. 3, pp. 457-468, 2011.

5. Y. Li and S. S. Ge, "Human-robot collaboration based on motion intention estimation," IEEE/ASME Transactions on Mechatronics, doi: 10.1109/TMECH.2013.2264533, 2013. 
6. J. J. Cabibihan, R. Pradipta, and S. S. Ge, "Prosthetic finger phalanges with lifelike skin compliance for low-force social touching interactions," Journal of NeuroEngineering and Rehabilitation, vol. 8, no. 1, p. 16, 2011.

7. S. S. Ge, C. H. Fua, and K. W. Lim, "Agent formations in 3D spaces with communication limitations using an adaptive q-structure," Robotics and Autonomous Systems, vol. 58, no. 4, pp. 333-348, 2010.

8. A. Billard, S. Calinon, R. Dillmann, and S. Schaal, "Robot programming by demonstration," in Springer Handbook of Robotics (B. Siciliano and O. Khatib, eds.), Springer, 2008.

9. H. Li, K. A. Lee, and B. Ma, "Spoken language recognition: From fundamentals to practice," Proceedings of the IEEE, vol. 101, no. 5, pp. 1136-1159, 2013.

10. L. Li, X. Yu, J. Li, G. Wang, J. Y. Shi, Y. K. Tan, and H. Li, "Vision-based attention estimation and selection for social robot to perform natural interaction in the open world," in Proceedings of the ACM/IEEE International Conference on Human-Robot Interaction (HRI), pp. 183-184, 2012.

11. L. Li, S. Yan, X. Yu, Y. K. Tan, and H. Li, "Robust multiperson detection and tracking for mobile service and social robots," IEEE Transactions on Systems, Man and Cybernetics, Part B, vol. 42, no. 5, pp. 1398-1412, 2012.

12. S. S. Ge, Y. Yang, and T. H. Lee, "Hand gesture recognition and tracking based on distributed locally linear embedding," Image and Vision Computing, vol. 26, no. 12, pp. 1607-1620, 2008.

13. R. Yan, K. P. Tee, Y. Chua, H. Li, and H. Tang, "Gesture recognition based on localist attractor networks with application to robot control," IEEE Computational Intelligence Magazine, vol. 7, no. 1, pp. 64-74, 2012.

14. N. Chen, C. M. Chew, K. P. Tee, and B. S. Han, "Human-aided robotic grasping," in Proceedings of the IEEE International Symposium on Robot and Human Interactive Communication (RO-MAN), pp. 75-80, 2012.

15. N. Chen, K. P. Tee, and C. M. Chew, "Assistive grasping in teleoperation using infra-red proximity sensors," in Proceedings of the IEEE International Symposium on Robot and Human Interactive Communication (RO-MAN), 2013. 